\title{
Deconfinement transition as a black hole formation by the condensation of QCD string
}

\author{
Masanori Hanada* \\ Stanford Institute for Theoretical Physics, Stanford University, Stanford CA 94305, USA \\ Yukawa Institute for Theoretical Physics, Kyoto University, Kitashirakawa Oiwakecho, \\ Sakyo-ku, Kyoto 606-8502, Japan \\ The Hakubi Center for Advanced Research, Kyoto University, Yoshida-Ushinomiya-cho, \\ Sakyo-ku, Kyoto 606-8501, Japan \\ E-mail: hanadadyukawa.kyoto-u.ac.jp
}

In the gauge/gravity duality, the deconfinement transition in the gauge theory is identified with the formation of a black hole in the dual gravity theory. By assuming this correspondence, many predictions on QGP have been made. In this talk, we justify this approach quantitatively, and also provide an intuitive way of understanding it. Firstly we give quantitative evidence for this identification from the thermodynamic study of the supersymmetric theory. We show that string theory and gauge theory give the same answer, even at finite temperature, including the $1 / N$ correction. Then we consider generic gauge theories and show that the deconfinement transition is the condensation of very long and self-intersecting QCD strings, which is analogous to the formation of a black hole in string theory.

The 32nd International Symposium on Lattice Field Theory,

23-28 June, 2014

Columbia University New York, NY

\footnotetext{
* Speaker.
} 


\section{Introduction}

In the gauge/gravity duality conjecture [W], the deconfinement phase of the gauge theory is dual to a black hole geometry in the gravity side [2]. In the first part of this talk, we give quantitative evidence for this identification, by solving a concrete example in both gauge and gravity sides, at the level of the string theory (i.e. finite $\alpha^{\prime}$ and finite $g_{s}$ ). In the second part, we give an intuitive way of understanding this correspondence, without referring to a sophisticated dictionary of the duality. Our argument does not assume the dual gravity description, and hence it is applicable to generic gauge theories including QCD.

\section{Quantitative test of the gauge/gravity duality}

Let us consider the $U(N)$ maximally supersymmetric Yang-Mills theory in flat $(p+1)$-dimensional spacetime ( $p=0,1,2$ and 3$)$, whose action is given by

$$
S=\frac{1}{g_{Y M}^{2}} \int d^{p+1} x \operatorname{Tr}\left\{\frac{1}{4} F_{\mu v}^{2}+\frac{1}{2}\left(D_{\mu} X_{i}\right)^{2}-\frac{1}{4}\left[X_{i}, X_{j}\right]^{2}\right\}+(\text { fermions }),
$$

where $\mu, v$ run from 1 to $p+1$ and $X_{i}(i=1,2, \cdots, 9-p)$ are scalar fields. In [B]], it has been conjectured that this theory is in the deconfining phase at any nonzero temperature, in the sense that the Polyakov loop has nonzero expectation value, and that it describes full string dynamics around the black $p$-brane. Near the horizon, the metric of the black $p$-brane geometry is given by

$$
\begin{aligned}
d s^{2}=\alpha^{\prime}\{ & \frac{U^{(7-p) / 2}}{g_{Y M} \sqrt{d_{p} N}}\left[-\left(1-\frac{U_{0}^{7-p}}{U^{7-p}}\right) d t^{2}+d y_{\|}^{2}\right] \\
& \left.+\frac{g_{Y M} \sqrt{d_{p} N}}{U^{(7-p) / 2}\left(1-\frac{U_{0}^{7-p}}{U^{7-p}}\right)} d U^{2}+g_{Y M} \sqrt{d_{p} N} U^{(p-3) / 2} d \Omega_{8-p}^{2}\right\}
\end{aligned}
$$

where the Yang-Mills coupling $g_{Y M}$ and the size of the gauge group $N$ in the corresponding super Yang-Mills theory are used. A constant $\alpha^{\prime}$ is the square of the string length, $\left(t, y_{\|}\right)$represent the $(p+1)$-dimensional extension of the brane, $U$ and $\Omega$ are the radial and angular coordinate of the transverse directions, and $U_{0}$ is the place of the horizon. The Hawking temperature is

$$
T_{H}=\frac{(7-p) U_{0}^{(5-p) / 2}}{4 \pi \sqrt{d_{p} g_{Y M}^{2} N}},
$$

where $d_{p}=2^{7-2 p} \pi^{(9-3 p) / 2} \Gamma((7-p) / 2)$. The string coupling constant is given by

$$
g_{s}=(2 \pi)^{2-p} g_{Y M}^{2}\left(\frac{d_{p} g_{Y M}^{2} N}{U^{7-p}}\right)^{\frac{3-p}{4}} .
$$

When $\lambda=g_{Y M}^{2} N$ is fixed, it behaves as $g_{s} \propto 1 / N$, in the same way as in 't Hooft's identification. The Hawking temperature and the mass of the black brane are identified with the temperature and the energy $E \equiv-\partial \log Z / \partial \beta$ of the gauge theory, where $Z$ is the partition function. 


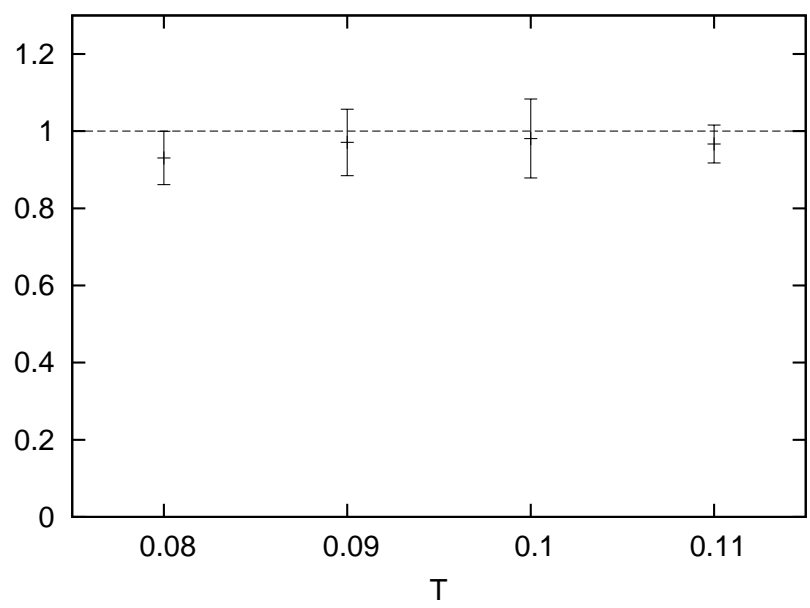

Figure 1: The coefficient of $1 / N^{2}$ divided by $-5.77 T^{0.4}$.

In this work we study the case of $p=0$. The gauge theory is quantum mechanics of $N \times N$ matrices, which was originally proposed as the matrix model of M-theory [四]. It can be studied extensively by using the Monte Carlo method. In the gravity side, the black hole mass can be calculated by adding stringy corrections to the black 0-brane geometry shown above. The result is [15]

$$
\frac{1}{N^{2}} E_{\text {gravity }}=\left(7.41 T^{2.8}+a T^{4.6}+\cdots\right)+\left(-5.77 T^{0.4}+b T^{2.2}+\cdots\right) \frac{1}{N^{2}}+O\left(\frac{1}{N^{4}}\right),
$$

where $T=\lambda^{-1 / 3} T_{H}$ is dimensionless effective temperature and $a, b$ are unknown constants. The energy $E_{\text {gravity }}$ is also made dimensionless, by multiplying $\lambda^{-1 / 3}$. In the following, we simply set $\lambda=1$ without loss of generality. Higher order terms in each power of $1 / N$ represent to the $\alpha^{\prime}$ corrections, which appear because strings are not point-like. If the gauge/gravity duality conjecture is correct, this expression must be reproduced from the matrix quantum mechanics.

The $O\left(N^{0}\right)$ terms of the dual gravity prediction (2.5]) have been tested previously. In particular, the $\alpha^{\prime}$ correction $a T^{4.6}$ has been confirmed with $a=-5.58(1)$, by looking at $T \gtrsim 0.5$ [目].

In order to study the $1 / N$ correction, we must study rather small values of $N$. Here we study $N=3,4$ and 5. (That we have to take $N$ small caused a technical problem which required a proper treatment; see [ [0].) We also have to study low temperature where the $\alpha^{\prime}$ correction becomes small, because there are too many fitting parameters otherwise. At $0.08 \leq T \leq 0.11$, we estimated the coefficient of $1 / N^{2}$ by using a fitting ansatz $E_{\text {gauge }}=7.41 T^{2.8}+c_{1}(T) / N^{2}+c_{2}(T) / N^{4}$ for each fixed value of $T$. We confirmed that $c_{1}(T)$ is consistent with $-5.77 T^{0.4}$; see Fig.W, in which this coefficient normalized by the dual gravity prediction, $c_{1} /\left(-5.77 T^{0.4}\right)$, is plotted. It is reasonably close to 1 . This is very strong evidence that the gauge/gravity duality holds at quantum gravity level, at least at the 1-loop level.

\section{Why the deconfinement phase describes the black hole}

Motivated by the numerical confirmation in Sec. 凤, in this section we give an intuitive expla- 
nation for the duality based on [ [ $]$ ] . As a concrete example, let us consider pure $U(N)$ Yang-Mills theory. The Hilbert space is spanned by Wilson loops acting on the vacuum $|0\rangle$, as $W_{C} W_{C^{\prime}} \cdots|0\rangle$. Here $W_{C}$ represent the Wilson loop along a closed contour $C$. In the standard identification of the Feynman diagrams and the string world-sheet [ $[$ ] , the Wilson loop is naturally interpreted as the creation operator of the string.

In the large- $N$ limit and at sufficiently strong coupling, the energy of the string is approximately proportional to its length. In the confinement phase, the energy is of order $N^{0}$ per unit volume, and hence a typical state is sparse gas of loops with finite length (Fig. ఇ, left). In this gas, two loops can intersect with each other and combined to form a longer string. Alternatively, when a loop intersects with itself, it can be split into two shorter loops. However such joining and splitting are suppressed at large- $N$.

In the deconfinement phase, the energy density is of order $N^{2}$. In this phase the loops necessarily intersect $O\left(N^{2}\right)$ times. Although the interaction at each intersection is $1 / N$-suppressed, small interactions at many intersections accumulate to a non-negligible amount. A standard entropic argument shows that typical state consists of finitely many very long and self-intersecting strings, whose lengths are of $O\left(N^{2}\right)$ (Fig. $\square$, right). In string theory, it is natural to interpret such very long and self-intersecting strings as a black hole [प, $\square]$. In this sense, the deconfinement transition can be understood as the formation of a 'black hole' through condensation of QCD strings.
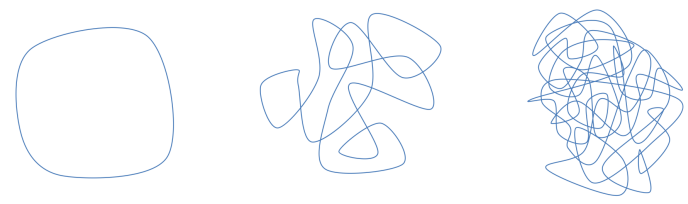

Figure 2: The confining phase is the gas of free strings (left). As the system is heated up, it becomes denser (middle), the interaction becomes non-negligible and a long string is formed (right). This is analogous to the formation of a black hole in string theory. [8]].

When we identify the long string with a black hole, fluctuations of the string near the horizon are regarded as open strings attached to black hole (Fig. (3)). In terms of the gauge theory, these open strings are open Wilson lines which have $N$ color degrees of freedom at their endpoints. Therefore, we can interpret that the black hole is made from $N$ D-branes.

In the Euclidean theory, the deconfinement is characterized by the condensation of the Polyakov loop, which can naturally be related to the black hole geometry in the gravity dual [2]. This can be explained from our picture as follows. First notice that the condensation of the Polyakov loop is 

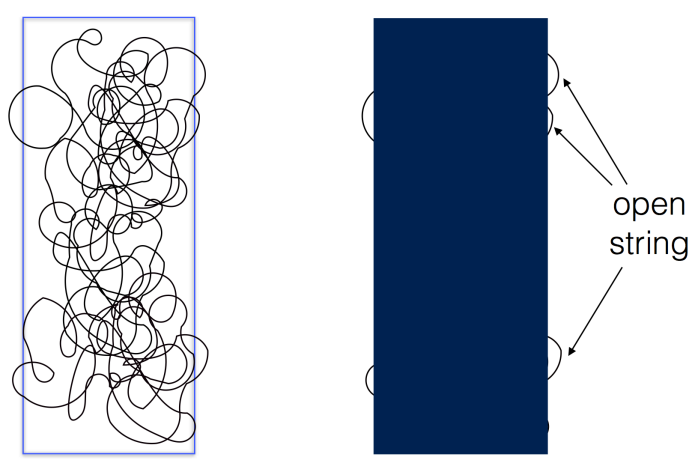

Figure 3: Closed string picture (left) and open string picture (right) [ [ ]].

equivalent to the disappearance of the linear confinement potential between a pair of probe quark and anti-quark. In terms of strings, the linear potential in the confining phase appears because an open string connecting probes must be stretched as they are separated. In the deconfining phase, however, as soon as a short open string is introduced, it intersects with closed strings many times, and they immediately interact at one of the intersections to form a long open string (Fig. 团). Therefore, probes can be separated without stretching the open string, or equivalently, without costing energy.

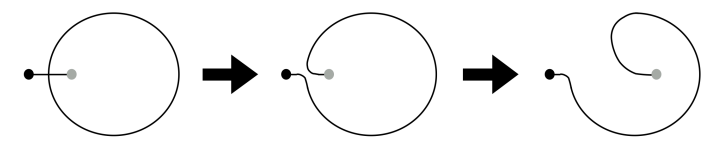

Figure 4: Deconfinement of a pair of probe quark and anti-quark [8].

In order to test this picture, we have analytically estimated the deconfinment temperature of the strong-coupling lattice gauge theory with $D$-dimensional spatial lattice and continuum time [ए2], and compared it with numerical simulation. Because the thermal transition in this model is of first order, we compared the analytically predicted critical temperature with the temperature region where the hysteresis of the expectation value of the Polyakov line is observed. The result is shown in Fig. [5. The vertical axis is $T / \lambda$, where $\lambda$ is the 't Hooft coupling, and the vertical axis is the spatial dimension $D$. The analytic prediction, $\left(T_{c} / \lambda\right)=1 /(2 \log (2 D-1))$, is shown by the dashed line. We can see $T_{c}$ is inside the hysteresis, which supports the validity of the analytic prediction and hence the long-string description of the deconfinement phase.

\section{Conclusion}

In the first part of this talk we have provided the first quantitative evidence for the gauge/gravity duality at finite temperature and finite- $N$. It means now lattice gauge theorists can study quantum gravity by studying the gauge theory. Motivated by this numerical confirmation, we proceeded to 


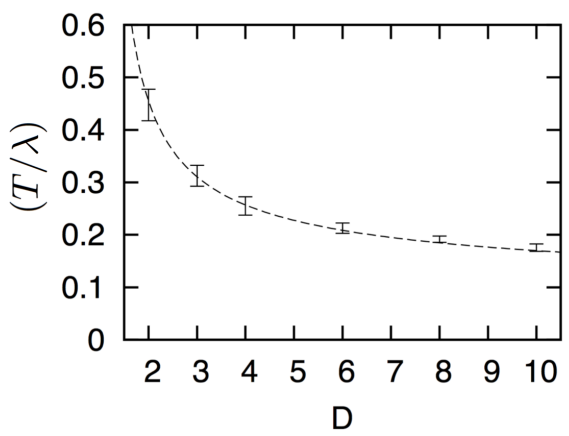

Figure 5: The temperature range of the hystereses of the Eguchi-Kawai model. The dashed line is the analytic prediction for the critical temperature, $\left(T_{c} / \lambda\right)=1 /(2 \log (2 D-1)) . N=64, n_{t}=16[8]$.

an intuitive way of understanding the duality in terms of QCD-strings. In the gauge theories with dual gravity descriptions, the condensation of the Wilson loops argued in this paper is equivalent to the condensation of fundamental strings and formation of an actual black hole. By following the time evolution of the loops, it should be possible to see how a black hole forms and thermalizes. As we have seen, qualitative features are common even in the strong coupling limit of simple matrix models, and hence they should serve as good toy models for a black hole. We believe that close collaboration between lattice gauge theorists and string theorists will be the key to the understanding of quantum gravity.

\section{Acknowledgments}

I would like to thank Y. Hyakutake, G. Ishiki, J. Maltz, J. Nishimura and L. Susskind for collaborations. I was supported in part by the Grant-in-Aid of the Japanese Ministry of Education, Sciences and Technology, Sports and Culture (MEXT) for Scientific Research (No. 25287046), and the National Science Foundation under Grant No. PHYS-1066293 and the hospitality of the Aspen Center for Physics.

\section{References}

[1] J. M. Maldacena, Adv. Theor. Math. Phys. 2, 231 (1998).

[2] E. Witten, Adv. Theor. Math. Phys. 2, 505 (1998).

[3] N. Itzhaki, J. M. Maldacena, J. Sonnenschein and S. Yankielowicz, Phys. Rev. D 58, 046004 (1998).

[4] T. Banks, W. Fischler, S. H. Shenker and L. Susskind, Phys. Rev. D 55, 5112 (1997).

B. de Wit, J. Hoppe and H. Nicolai, Nucl. Phys. B 305, 545 (1988).

[5] Y. Hyakutake, PTEP 2014, no. 3, 033B04.

[6] M. Hanada, Y. Hyakutake, J. Nishimura and S. Takeuchi, Phys. Rev. Lett. 102, 191602 (2009).

[7] M. Hanada, Y. Hyakutake, G. Ishiki and J. Nishimura, Science 344, 882 (2014).

[8] M. Hanada, J. Maltz and L. Susskind, arXiv:1405.1732 [hep-th]. 
[9] G. 't Hooft, Nucl. Phys. B 72, 461 (1974).

[10] L. Susskind, In *Teitelboim, C. (ed.): The black hole* 118-131.

[11] G. T. Horowitz and J. Polchinski, Phys. Rev. D 55, 6189 (1997).

[12] J. B. Kogut and L. Susskind, Phys. Rev. D 11, 395 (1975). 